\title{
A HERO for Stroke Patients: a new hand exoskeleton 3D printed on textiles for rehabilitation
}

Rommel Soares de Araujo ( $\square$ a.rommelsoares@gmail.com )

Santos Dumont Institute https://orcid.org/0000-0001-8509-2191

Camille Reategui Silva

Santos Dumont Institute

Severino Peixoto Nunes Netto

Santos Dumont Institute

Edgard Morya

Santos Dumont Institute

Fabricio Lima Brasil

Santos Dumont Institute

\section{Methodology}

Keywords: hand exoskeleton, rehabilitation, post-stroke, 3D printing, textiles

Posted Date: September 24th, 2020

DOI: https://doi.org/10.21203/rs.3.rs-72828/v1

License: (c) (1) This work is licensed under a Creative Commons Attribution 4.0 International License. Read Full License 


\title{
A HERO for Stroke Patients: a new hand exoskeleton 3D printed on textiles for rehabilitation
}

\author{
Rommel Soares de Araujo ${ }^{*}$, Camille Reategui Silva, Severino Peixoto Nunes Netto, Edgard Morya and \\ Fabricio Lima Brasil
}

\footnotetext{
${ }^{*}$ Correspondence:

a.rommelsoares@gmail.com

Edmond and Lily Safra

International Institute of

Neuroscience. Santos Dumont

Institute, 1560 Santos Dumont

Ave., Countryside, 59280-000

Macaiba, Brazil

Full list of author information is

available at the end of the article
}

\begin{abstract}
Background: Stroke survivors can be affected by motor deficits in the hand. Robotic equipment associated with brain-machine interfaces (BMI) may aid the motor rehabilitation of these patients. BMls involving orthotic control by motor imagery practices have been successful in restoring stroke patients' movements. However, there is still little acceptance of the robotic devices available, either by patients and clinicians, mainly because of the high costs involved. Motivated by this context, the present work aims to design and construct the Hand Exoskeleton for Rehabilitation Objectives (HERO) to recover extension and flexion movements of the fingers.
\end{abstract}

Methods: A 3D printing technique in association with textiles was used to produce a lightweight and wearable device. 3D-printed actuators have also been designed to reduce equipment costs. The actuator transforms the torque of DC motors into linear force transmitted by Bowden cables to move the fingers passively. The exoskeleton was controlled by neuroelectric signal electroencephalography (EEG). Concept tests were performed to evaluate control performance. A healthy volunteer was submitted to a training block with the exoskeleton, according to the $\mathrm{Graz}-\mathrm{BCl}$ protocol. Ergonomy was evaluated with a 2D tracking software.

Results: The outcome of the applied manufacturing technique was aesthetically pleasing. HERO's glove can be compared to ordinary clothing. The weight over the hand was around $102 \mathrm{~g}$. The volunteer was able to control the exoskeleton with $91.5 \%$ accuracy.

Conclusions: HERO's project resulted in a lightweight, simple, portable, ergonomic, and low-cost device. Its use is not restricted to a clinical setting. Thus, users will be able to execute motor training with the HERO at hospitals, rehabilitation clinics, and home, increasing the rehabilitation intervention time. This may favor motor rehabilitation and improve the life quality of stroke survivors.

Keywords: hand exoskeleton; rehabilitation; post-stroke; 3D printing; textiles

\section{Background}

Most of the Activities of Daily Living (ADLs) directly involve hand motor skills. However, a serious but common disease impairs persistently arm and hand function: the stroke. In 2017, an estimated 11.9 million people worldwide suffered strokes, representing an increase of $21 \%$ in ten years from 2008 [1] in one of the main causes 
of long-term disabilities [2]. Six months after the stroke onset, $30 \%$ of hemiplegic stroke patients remain with no arm and hand function. This situation is consistent with considerable damage to the corticospinal tract [3], which unable the patient to perform basic hand movements such as cylindrical and pinch grasps movements.

A proven effective strategy to mitigate or reverse this picture is the ConstraintInduced Movement Therapy (CIMT), which stimulates the patient to "relearn" to use the impaired limb in ADLs by restricting the movements of the healthy arm/hand $[4,5,6]$. Despite its effectiveness, the CIMT fail to engage a large number of patients, mainly due to factors such as the restriction of movement for long periods, the immobilization devices [7], and the requirement of some residual motor skills in affected limbs $[4,8]$. Therefore, severely hemiplagic stroke survivors are not able to benefit from this therapy.

Robotic devices based on Brain-Machine Interface (BMI) are a promising tool for post-stroke rehabilitation. BMI encompasses technologies to communicate the brain with a computer or a machine by capturing electrical, magnetic or chemical activities of the brain [9]. In the last few years, the advance of new BMI technologies and pioneering results attracted researchers attention to developing robotic devices to recover hand movements.

In a broad study [10], 32 chronic stroke patients with paralysis and no extension movement in one hand underwent a daily training with an EEG controlled orthotic device plus behaviorally oriented physiotherapy for one month. Patients of the experimental group, improved significantly arm and hand movements. In addition, recent studies show that trying to rehabilitate the hand can improve not only the hand but also arm skills [11].

\section{Hand Exoskeletons}

Exoskeletons for hand rehabilitation eventually gained the researchers' attention due to their proposal of being a limb extension. If one project is capable of developing a lightweight, practical, ergonomic, portable, and mainly functional device, one can expect that patients will be more likely to engage in rehabilitation interventions or even take the device to train at home. That way, stroke patients could leave the clinical setting and increase the therapy intensity. Additionally, the device could also assist the patients in several ADLs.

It is well known that users' requirements, e.g., weight, comfort, aesthetics, and cost are directly related to the success or failure of hand rehabilitation devices [12]. Some researches [13] suggest that the maximum weight of the exoskeleton over the hand should not exceed $500 \mathrm{~g}$. In a review by [14], all exoskeletons surveyed weighed less than $285 \mathrm{~g}$ over the hand. Actuators and control systems can be attached to the body and should weight less than $3 \mathrm{~kg}$, the average weight of portable electronic devices, e.g., laptops [15].

Furthermore, cost is also one of the major problems faced by designers. High prices may cause patients to seek only conventional treatments, such as medicines and physical therapy. Moreover, the acquisition of such devices may be unfeasible for intensive training within patients' homes. Seeking to solve these problems, several studies have proposed different exoskeletons specifications. They vary as to the total degrees of freedom (DOF), types of mechanisms and actuators, which may 
be electric, pneumatic, cable-driven or shape-memory alloys [14]. Different manufacturing techniques were also applied, with emphasis on 3D printing [12], a fast, practical and low-cost prototyping technique, which allows manufacturing of complex structures that could not, or would be very expensive, to be produced by other traditional techniques, e.g., machining.

\section{Soft Robotics}

Soft Robotics aims to developing ergonomic and biocompatible devices by trying to mimic the biomechanics of living beings. This technology is interesting and suitable to develop new hand exoskeletons because it favors the design of mechanisms with no need to incorporate rigid parts to their projects. Thus, it is possible to develop more friendly devices with anatomic-like functionalities. In a review by [14], most of the previously developed soft exoskeletons were driven by pneumatic actuators (64\%). In sequence were cable-driven devices (34\%) and only a small part was driven by hydraulic actuators $(2 \%)$.

Although pneumatic systems can perform movements similar to natural movements of the hand [14], the pumps to compress the air are usually bulky and noisy. Therefore, they end up compromising portability, and daily live use [16]. Even though current devices may be quieter, pneumatic systems are not capable to generate great efforts. Hydraulic actuators follow the same principle, however, the working fluid is a liquid [15] and, thus, they are usually heavier than other actuators [14].

In cable-driven exoskeletons, the transmission of force generated by the actuators is accomplished by Bowden cables. Usually made of steel, they are commonly found in automotive and aeronautical industries. These cables have the ability to both pull and push mechanical components even though the path between the actuator and the component is curved. The main advantage of using Bowden cables to drive exoskeletons is because this configurations allows placing the actuators in a separate module, reducing the weight over the hand $[17,18,19]$.

\section{D printing}

3D printing technology has evolved significantly over the years. At first, 3D printers were used to rapidly prototype initial models to test and adjust specific design details. Currently, it is already possible to find functional 3D printed devices aiming to lower costs and to speed up product customization. Combining soft robotics to 3D printing can, thus, be a solution to the main problems of developing a rehabilitation device for the hand [12].

One of the most widespread, versatile, and accessible 3D printing techniques is the Fused Deposition Modeling (FDM), in which a material - typically a polymer - is fused and extruded onto a surface layer by layer. The molten material of a new layer adheres to the previous layers. We observed that this technique permits one to pause printing to insert porous textiles and then resume printing to form a "fabric sandwich". Thus, the new layers of molten material are free to adhere to other layers through the pores, enabling the fabrication of wearable devices.

The authors are not aware of any study applying this technique to manufacture hand exoskeletons. Searches on specialized and social media only show applications 
for entertainment and fashion, e.g., cosplay costumes. In academics, as far as we are aware, only two studies published in proceedings quantitatively analyzed 3D printings with embedded textiles [20, 21]. According to [20], PLA filaments have greater adhesion strength. Both the width of the mesh opening and the thickness of the fabrics directly influence the adhesion of the PLA.

Addressing these challenges, we decided to develop the HERO - Hand Exoskeleton for Rehabilitation Objectives. An exoskeleton built with PLA-based 3D printed parts embedded with porous fabrics. A new actuator mechanism was also developed — in order to reduce costs - using the same technique to drive Bowden cables to passively move the fingers in a natural pattern. The HERO was controlled by EEG signals and conceptually tested with a healthy subject undergoing motor imagery (MI) paradigms, i.e., the imagination of a movement without activating any muscle.

\section{Methods}

\section{Exoskeleton's design}

HERO's 3D design was drawn on SolidWorks $2013^{\circledR}$. All the 3D parts were aligned in a glove shape to a Cartesian plane, simulating a $3 \mathrm{D}$ printer table. The pieces were expected to attach to the fabric during printing and retain the glove format. At first, the dimensions used to align the pieces were specified by measurements of a designer hand. Further on, end-user hand measurements should be used to customize the exoskeleton. The HERO's design is presented in Figure 1.

\section{Manufacture}

FDM technique with PLA filament was used to prototype the hand exoskeleton. PLA is a biodegradable polymer composed of polylactic acid molecules whose manufacturing process uses renewable materials, such as carbohydrates from corn, potatoes or dairy products [22]. Besides, PLA filaments result in prints with better finishing and geometric tolerances [23].

Three different fabrics were previously tested. All of them are commercially known as tulle, a lightweight and resistant netting most commonly used to make bridal veils. Figure 2 shows the chosen tulles. They are composed mainly by polyester but vary in structure, color and elasticity. The $\mathrm{T} 1$ and $\mathrm{T} 2$ samples have similar mesh openings and thickness, although they have different colors. T3 sample was chosen because of its larger hexagonal mesh compared to T1 and T2. T3 is thicker and less elastic than other samples.

Printing tests were developed in each fabric with some parts of the exoskeleton. Printing parameters were: print speed of $50 \mathrm{~mm} / \mathrm{s}$, infill density of $70 \%$, layer high of $0.2 \mathrm{~mm}$, extruder temperature of $211^{\circ} \mathrm{C}$, heated bed temperature of $65^{\circ} \mathrm{C}$, and no support. G-code was adapted inserting a pause code M25 followed by the G1 Z100 code to raise the nozzle $100 \mathrm{~mm}$ after completing the third layer. After that, the fabric was attached to the table with paper clips and duct tape (see Figure 3A). Printing was resumed manually then.

Figures 3B, 3C, and 3D show amplified pictures of the printing tests. All the parts printed was firmly attached to the tulles. PLA layers were observed surrounding fabric fibers. Attempts to separate the parts from the fabric revealed no tendency for delamination between the intersection layers with the fabric. 
$\mathrm{T} 1$ and T2 were similarly strong. Both $\mathrm{T} 1$ and $\mathrm{T} 2$ seemed more resistant than T3. T1 sample was chosen due to its resistance and to its color similar to the skin. Based on the test results, two pieces of $\mathrm{T} 1$ were inserted between layers 3-4, and layers 6-7 to improve the exoskeleton's mechanical resistance.

\section{Actuator's design}

HERO's actuation system was designed to be attached to the patient's healthy leg. A cable-driven system was chosen to favor device portability and lower complexities. These systems commonly use linear servo motors to pull and push Bowden cables. However, this configuration requires a mechanism similar to the described in Figure 4 to keep the cable always restricted to a wrapped area preventing non-desired flexions under compression. Thus, if a actuator stroke of $x \mathrm{~mm}$ is required, one would also need a $x \mathrm{~mm}$ mandrel resulting in a final length of $2 x \mathrm{~mm}$, not dimensionally efficient.

Thus, a new actuator was developed using a concertina-like mechanism to drive HERO's movements with the same manufacture methodology of the exoskeleton (see Figure 5). This mechanism limits a maximum gap where the cable can pass through under compression without flexing. Figure 6 shows the actuator's 3D assembly model.

Each actuator is capable of moving two cables at the same time. The maximum stroke is $60 \mathrm{~mm}$. To move all the five fingers, two actuators were required. An extra actuator was designed to move only the thumb. The actuator has a principle similar to ordinary linear actuators in which a lead screw driven by a DC motor moves a nut to apply force to pull or to push the cables according to the rotation direction. All nuts required for the project were inserted during 3D printing. A honeycomb structure was applied to the actuator base to reduce weight.

\section{Actuator modeling}

Typical ADLs, such as manipulating small objects with pinch and palmar grips, usually require forces up to $20 \mathrm{~N}$ at the fingertips [24]. Studies regarding post-stroke pathological conditions of the hand have shown that torques of $0.9 \mathrm{~N} \cdot \mathrm{m}$ applied to the metacarpophalangeal joint are enough to extend the four fingers (except thumb) of patients with severe spasticity in flexor muscles [25, 26]. Therefore, it was established that a criteria of at least a $0.25 \mathrm{~N} \cdot \mathrm{m}$ torque per finger should be applied to the metacarpophalangeal joint.

Considering the finger as a rigid body and a lever arm equal to $10 \mathrm{~mm}$ between the cable attachment point and the joint (as indicated in Figure 7), it is assumed that the momentum generated by the cable tension should be equal to the momentum applied to the joint. This leads to a $T$ tension of $25 \mathrm{~N}$.

However, friction also needs to be considered as it influences directly Bowden cables efficiency. Friction depends on materials of both cable and conduit as well as on the curvature angle between the actuator and the fixation point [27]. According to [28], the required tension must be calculated by Equation 1 to account the friction.

$$
T_{\text {req }}=T e^{\mu \theta}
$$


Where $T_{\text {req }}$ is the actuator required force, $\mu$ is the coefficient of dynamic friction between the cable and the conduit, and $\theta$ is the curvature angle of the cable in radians. A $15.0 \mathrm{~N}$ friction force was calculated (37.6\% of $\left.T_{\text {req }}\right)$, considering a tension criteria of $T=25 \mathrm{~N}$, a coefficient of friction of $\mu=0.150$ - according to [28] — and a curvature angle of $\pi$ radians between the actuator on the leg and the exoskeleton on the hand. Therefore, the required tension per finger was $T_{\text {req }}=40.0 \mathrm{~N}$.

A required DC motor torque $\tau_{\text {req }}$ of $0.055 \mathrm{~N} \cdot \mathrm{m}$ was calculated with Equation 2, considering a M6x1 lead screw lubricated with machine oil [29]. Based on these specifications, a Ningbo Leison (Ningbo, China) DC motor with reduction box was chosen. An Arduino Uno and an Arduino shield VNH2SP30 full bridge motor driver controlled DC motors rotation. Micro switches were coupled to the actuator base to indicate stroke limits.

$$
\tau_{\mathrm{req}}=\frac{F d_{\mathrm{m}}}{2}\left(\frac{l+\pi f d_{\mathrm{m}} \sec \alpha}{\pi d_{\mathrm{m}}-f l \sec \alpha}\right)
$$

Where $F$ is the axial force, i.e., the same as $T_{\text {req }}, d_{\mathrm{m}}$ is the pitch diameter of the threaded bar, $l$ is the thread pitch, $f$ is the coefficient of dynamic friction between the nut and the bar, and $\alpha$ is half the thread angle.

\section{Actuators mechanical assessment}

Tests were performed to access actuators mechanical capacity. The cable was coupled to a load cell that transmitted force data to an Arduino Uno. The DC motor was supplied with voltages ranging from 3 to $12 \mathrm{~V}$ in steps of $1 \mathrm{~V}$. The maximum pull force was measured three times for each voltage.

\section{HERO's control}

An EEG-based BMI was implemented to control HERO's movements. Native scenarios of the OpenVibe (OV) software, an open-source platform to process biological signals, were used. These scenarios carry out the Graz-BCI paradigm, widely used in academics to allow human-computer interaction through MI training [30]. In this case, OV algorithms had to detect two classes. Class 1 (C1), an arrow appears in the screen to the left side: the subject relaxes. Class $2(\mathrm{C} 2)$, an arrow appears in the screen to the right side: the subject performs the right hand MI, without any movement. Whenever the OV detects a class, a specific stimulus is triggered.

The OV scenarios were adapted by adding a "Button VRPN server" box to communicate the software with the actuators' control system. This box creates servers that share data with external devices by exposing boolean virtual button states (on/off) through a Virtual Reality Peripheral Network (VRPN). Three buttons were configured in the box. The first and the second buttons turned on whenever a $\mathrm{C} 1$ and $\mathrm{C} 2$ stimulus occurred, respectively. Button 3 only turned on to indicate the beginning of a new trial.

The VRPN libraries was compiled in Microsoft Visual Studio 2013 ${ }^{\circledR}$ (MVS 2013). A $\mathrm{C}++$ program was developed to ensure that OV buttons output data were transferred via USB port to the Arduino Uno. Therefore, the program sent values "0", 
"1" or " 2 " whenever buttons 1, 2, or 3 were turned on, respectively. More information about this program is available in [31].

A command to push the cables ran each time the value "1" was received by the Arduino Uno, passively flexing the fingers as long as button 2 remained pressed. Motors were braked if the value " 0 " was detected. At the beginning of each new trial, the Arduino received the value " 2 " and repositioned the actuator to the beginning of the stroke.

Proof of concept

A proof of concept was develop to test HERO's performance. One right-handed healthy subject was invited to use the HERO under approval of an Ethics Committee (No. 79649717.0.0000.529). The subject — naive to BMI training — underwent a section consisting of a MI training block and an experimental block with online feedback. Each block consisted of a total of 20 trials of $\mathrm{C} 1$ and 20 trials of C2 randomly assigned.

\section{EEG acquisition}

Brain signal capture was performed with the 16 channel ActiCAP ${ }^{\circledR}$ (Brain Products GmbH, Germany). V-AMP ${ }^{\circledR}$ amplifier (Brain Products GmbH, Germany) was used in association with the ActiCAP. The data was acquired at $512 \mathrm{~Hz}$. Wet electrodes were positioned in a way to encompass the motor cortex region. The chosen electrodes positions were: FC3, C5, C3, C1, CP3, P3, Fz, Cz, FC4, C2, C4, C4, $\mathrm{CP} 4, \mathrm{P} 4, \mathrm{CPz}$ and $\mathrm{Pz}$ according to international standard 10-10. The FCz electrode was used as reference and the AFz electrode as ground. A bandpass filter between 8 and $30 \mathrm{~Hz}$ was applied.

\section{Ergonomic evaluation}

Natural grasp movements of the subject was compared to the passive movements developed by HERO. The fingers trajectories were calculated by tracking a white dot positioned on the index fingertip with the help of Kinovea software. Trajectories were averaged and compared.

\section{Results}

Figure 8 shows the HERO's glove prototype printed with translucent PLA and T1 fabric after cutting the fabric and adding fixing elastics. This configuration achieved the best aesthetic design. The glove material-only costs were less than US\$ 1 [see Additional file 1].

Three actuators were responsible for flexing/extending all five fingers. A two-wire actuator was configured with a $50 \mathrm{~mm}$ stroke to drive the index and little fingers. Another two-wire actuator was configured with a $60 \mathrm{~mm}$ stroke to drive the middle and ring fingers. Finally, a $40 \mathrm{~mm}$ stroke one-wire actuator was responsible for driving the thumb. Each actuator weighed about $240 \mathrm{~g}$.

The mean force (in kgf) curve is presented in Figure 9 with their respective standard deviations. The relationship between the maximum actuator force and the voltage supply presented a linear behavior. The peak value was $8.35 \mathrm{kgf}$, equivalent to $81.9 \mathrm{~N}$ when supplied with $12 \mathrm{~V}$. 
HERO's final assembly

The exoskeleton and the actuator were connected by inexpensive cables and conduits. Figure 10 shows HERO assembled to the body. Actuators were fixed to the leg and conduits were hidden by the user's clothes. Elastic bands were attached to the forearm to hold the cables and to give greater mobility. The exoskeleton weighed around $102 \mathrm{~g}$ after mounted over the hand, which corresponds to only $20 \%$ of the maximum weight suggested by [13]. The weight of the control system plus the actuators was less than half of the maximum weight requirement [15], or, more specifically, $1.4 \mathrm{~kg}-588 \mathrm{~g}$ of battery, $129 \mathrm{~g}$ of the Arduino Uno, motor shield, and connection cables, and $683 \mathrm{~g}$ of actuators.

Proof of concept

Control performances

The naive subject was able to control the HERO with an accuracy of $91.5 \%$. The variation between trials directly influenced the HERO's control. Trials in which the algorithm was unable to accurately predict the correct class led to intermittent passive movements. This situation can be observed in Figure 11. This figure shows the values sent to the Arduino Uno in different trials. HERO's actuators were activated only when the Arduino received the value "1".

The upper graph represents a low-performance trial. In this case, the actuators were activated for short periods, and the exoskeleton was not able to reach the maximum movement range at the end of the trial. In trials with higher performances - lower graph - the actuators had enough time to reach their maximum stroke, and, therefore, full flexion was achieved.

Ergonomic evaluation

Figure 12 describes the trajectory of the index fingertip during finger flexion movements in comparison of 2D-trajectories of active - natural - and passive movements - performed by the exoskeleton.

\section{Discussion}

Manufacture methodology

In this work, for the first time, an innovative manufacturing method was applied within the scope of assistive technologies and Soft Robotics devices to make a low cost and lightweight hand exoskeleton for stroke rehabilitation. The fabricassociated FDM technique provided a rapid and versatile HERO prototyping.

HERO's final prototype is aesthetically pleasing. The combination of the transparent PLA with the invisible tulle gives the impression that the exoskeleton makes part of the hand. This aesthetic aspect is one of the key features to consider when designing robotic devices for rehabilitation [12]. The HERO's low-cost design may contribute to better acceptance by stroke patients and may motivate intensive hand rehabilitation training and possible use during ADLs.

FDM technique had several advantages, such as good aesthetic finishing, precision in dimensional tolerances, rapid and versatile prototyping, and easy replacement of parts. The counterpoints were: the necessity of constant printer maintenance to guarantee high-quality parts and uncertainties about using this methodology to mass production. 
HERO design used dimensional tolerances of a $0.3 \mathrm{~mm}$ gap for clearance fits and a $0.15 \mathrm{~mm}$ gap for transition fits. These values are in line with previous work [23]. However, factors such as incorrect table calibration, and ambient and extruder temperature variations directly affect the quality of the joints. Nevertheless, this work showed that functional parts can be produced by the FDM technique, contributing to the findings of previous works $[32,33]$.

\section{Durability}

Although HERO's design is aesthetically pleasing, the durability of the "fabric sandwich" during intensive use is not yet known. The actuator also needs to be evaluated in this regard. Therefore, extensive testing still needs to be performed. Design changes such as part resizing, different fabrics configurations, and also the application of seams could increase prototype durability.

\section{Mechanical specifications of actuators}

In this study, a torque criterion was adopted based on previous works [25, 26]. According to these studies, the specified torque is enough to apply HERO in ADLs and in clinical procedures with spastic stroke patients. This criterion was higher than those generally adopted in exoskeleton projects [34, 35]. However, thorough analyzes still need to be done to assess the real force applied to the fingertips and to overcome spasticity in stroke patients.

\section{Weight}

The weight of the hand-mounted exoskeleton increased from $58 \mathrm{~g}$ to about $102 \mathrm{~g}$ by adding cables and conduits. Nevertheless, it is only about $20 \%$ of the $500 \mathrm{~g}$ maximum criterion suggested by [13]. It is also below the average weight of cabledriven exoskeletons [14]. The control system weighted about $1.4 \mathrm{~kg}$. Although this value is within the initial specification of $3 \mathrm{~kg}$ [15], many adjustments still can be done such as the use of lighter batteries. It is noteworthy that the mentioned values do not consider the BMI apparatus.

\section{Cost}

The material-only costs were equivalent to US\$ 125.00 (one hundred and twenty five dollars) [see Additional file 1].

\section{Ergonomics}

The passive motion performed by the HERO was compared to the natural handgrip movement. HERO was able to impose a trajectory very close to a natural movement. Although the HERO's mechanical system is not able to perform complex movements, studies suggest that increasing the DOF may not be the best alternative for post-stroke rehabilitation. One of the reasons is that most patients need to recover simple movements before performing more precise movements. In addition, more complex devices involve equally complex control techniques, which may reduce patient enrollment. 


\section{EEG-based BMI}

EEG is an interesting alternative for total limb paralyzed stroke patients. However, robust EEG equipment is expensive. Although low-cost devices such as Emotiv Epoc are already available, the signal quality of these devices is questionable, and the use is now based on rental [12].

Alternatively, there are cheaper do-it-yourself (DIY) EEG devices where users can build and test their own EEG [36]. These devices have already been used in BMI applications and could be associated with HERO. However, despite the efforts of DIY EEG designers, aspects such as safety, durability, and reliability are still questionable and not properly specified.

The control accuracy of BMI was high for a naive subject, and it can increase with regular training. This high decoding control rate could be interesting for the patient's rehabilitation, even though it's not related to the HERO's design and functionality.

Also, [37] suggests that most stroke survivors are still able to control devices by residual myoelectric activity. Thus, it is possible that human-machine interfaces (HMIs) such as EMG could be associated with the HERO for motor rehabilitation [38]. This would considerably decrease the total costs involved in rehabilitation interventions and facilitate both clinical and intensive use in patients' homes.

\section{Limitations}

Although the required momentum to move the fingers of patients with severe spasticity was taken into account, only a proof of concept was performed with a healthy subject. No tests have been performed on real patients to assess the usability and the acceptability of the HERO. Neither can be inferred whether the HERO's EEGbased training can bring clinical benefits to chronic stroke patients such as happened in [10]. Future clinical studies may provide appropriate answers to these questions. Besides, safety mechanisms have yet to be implemented.

\section{Conclusion}

Development of robotic devices for post-stroke hand rehabilitation has made rapid progress, especially over the last ten years. Although the neurophysiological basis behind motor recovery is not fully comprehended, few weeks of BMI therapy improves significantly motor control.

Here, an innovative and low-cost robotic device for hand rehabilitation after stroke has been proposed. The mechanical design was based on a large literature review. HERO's design tried to balance different parameters, such as aesthetics, comfort, practicality, ergonomics, portability and low-cost.

A differential of this project is its manufacturing methodology. Combining fabrics to $3 \mathrm{D}$ printed robotic parts may be an alternative to develop new wearable devices for healthcare. Besides, simple and low-cost parts were used to build the HERO's electrical and mechanical systems guaranteeing easy maintenance. The result was a lightweight, simple, portable, and inexpensive device.

A large scale test still needs to be developed. It is expected that HERO's training sessions can be performed not only in hospitals and clinical settings but also in patients' homes enabling intensive rehabilitation training and higher movement 
recovery for stroke patients, or patients with movement disorders. Another possibility is to use the HERO to assist patients in various ADLs, which may increase the quality of life of not only stroke patients but also their caregivers.

Ethics approval and consent to participate

One healthy participant signed written consent prior to participation. This study has been approved by the Ethics Committee of the Universidade Potiguar (No. 79649717.0.0000.529).

Consent for publication

The volunteer provided permission for collecting and publishing the data by a signed consent after a proper explanation about the objectives, importance, and the way data would be collected in this study.

Availability of data and materials

The datasets during and/or analyzed during the current study are available from the corresponding author on reasonable request.

Competing interests

The authors declare that they have no competing interests.

Funding

This study was funded by the National Council for Scientific and Technological Development (CNPq), and supported by institutional funds provided by the Graduate Program in Neuroengineering of the Edmond and Lily Safra International Institute of Neuroscience, Santos Dumont Institute.

\section{Author's contributions}

Rommel S. Araujo conceived of the present idea, design, analytic calculation, data collection and analysis, study concept and design, and wrote the manuscript with support of Fabricio L. Brasil. Camille R. Silva contributed with the HERO's serial communication with the Arduino Uno and assisted with other technical details. Severino P. N. Netto enabled the access to the 3D printers from the Laboratory of Technological Innovation in Health (LAIS), assisted with technical details and revision of the final manuscript. Edgard Morya supervised the project and helped with material aquisition. Fabricio L. Brasil, contributed to the study concept and design, critical revision of the manuscript for important intellectual content, obtained funding, and supervised the project.

\section{Acknowledgements}

The authors would like to thank the Coordination for the Improvement of Higher Education Personnel (CAPES) and the National Council for Scientific and Technological Development (CNPq) from the Brazilian government for its financial support on the project (number 428,699/2016-2), the Laboratory of Technological Innovation in Health (LAIS), especially Dr. Danilo Pinto Alves Nagem for helping with the 3D printers, Gabriela de Araujo Albuquerque and Julia Apolonio for the help of 3D printing and valuable comments regarding the project, and the Graduate Program in Neuroengineering of the Edmond and Lily Safra International Institute of Neuroscience, Santos Dumont Institute.

\section{References}

1. Global Burden of Disease Collaborative Network. Global Burden of Disease Study 2017 (GBD 2017) Results. Institute for Health Metrics and Evaluation (IHME), Seattle, United States (2018).

http://ghdx.healthdata.org/gbd-resultstool

2. Benjamin, E.J., Virani, S.S., Callaway, C.W., Chamberlain, A.M., Chang, A.R., Cheng, S., Chiuve, S.E., Cushman, M., Delling, F.N., Deo, R., et al.: Heart disease and stroke statistics-2018 update: a report from the american heart association. Circulation 137(12), 67 (2018)

3. Kwakkel, G., Kollen, B.J., van der Grond, J., Prevo, A.J.: Probability of regaining dexterity in the flaccid upper limb: impact of severity of paresis and time since onset in acute stroke. Stroke 34(9), 2181-2186 (2003)

4. Wolf, S.L., Winstein, C.J., Miller, J.P., Taub, E., Uswatte, G., Morris, D., Giuliani, C., Light, K.E., Nichols-Larsen, D.: Effect of constraint-induced movement therapy on upper extremity function 3 to 9 months after stroke: the excite randomized clinical trial. Jama 296(17), 2095-2104 (2006)

5. Langhorne, P., Bernhardt, J., Kwakkel, G.: Stroke rehabilitation. The Lancet 377(9778), 1693-1702 (2011)

6. Pollock, A., Farmer, S.E., Brady, M.C., Langhorne, P., Mead, G.E., Mehrholz, J., van Wijck, F.: Interventions for improving upper limb function after stroke. Cochrane Database Syst Rev 11(11) (2014)

7. Page, S.J., Levine, P., Sisto, S., Bond, Q., Johnston, M.V.: Stroke patients' and therapists' opinions of constraint-induced movement therapy. Clinical rehabilitation 16(1), 55-60 (2002)

8. Taub, E., Uswatte, G., Pidikiti, R., et al.: Constraint-induced movement therapy: a new family of techniques with broad application to physical rehabilitation-a clinical review. Journal of rehabilitation research and development 36(3), 237-251 (1999)

9. Birbaumer, N., Cohen, L.G.: Brain-computer interfaces: communication and restoration of movement in paralysis. The Journal of physiology 579(3), 621-636 (2007)

10. Ramos-Murguialday, A., Broetz, D., Rea, M., Läer, L., Yilmaz, Ö., Brasil, F.L., Liberati, G., Curado, M.R. Garcia-Cossio, E., Vyziotis, A., et al.: Brain-machine interface in chronic stroke rehabilitation: a controlled study. Annals of neurology 74(1), 100-108 (2013)

11. Balasubramanian, S., Klein, J., Burdet, E.: Robot-assisted rehabilitation of hand function. Current opinion in neurology 23(6), 661-670 (2010)

12. McConnell, A., Moioli, R., Brasil, F., Vallejo, M., Corne, D., Vargas, P., Stokes, A.: Robotic devices and brain-machine interfaces for hand rehabilitation post-stroke. J Rehabil Med 49, 449-460 (2017) 
13. Aubin, P.M., Sallum, H., Walsh, C., Stirling, L., Correia, A.: A pediatric robotic thumb exoskeleton for at-home rehabilitation: the isolated orthosis for thumb actuation (iota). In: Rehabilitation Robotics (ICORR), 2013 IEEE International Conference On, pp. 1-6 (2013). IEEE

14. Chu, C.-Y., Patterson, R.M.: Soft robotic devices for hand rehabilitation and assistance: a narrative review. Journal of neuroengineering and rehabilitation 15(1), 9 (2018)

15. Polygerinos, P., Wang, Z., Galloway, K.C., Wood, R.J., Walsh, C.J.: Soft robotic glove for combined assistance and at-home rehabilitation. Robotics and Autonomous Systems 73, 135-143 (2015)

16. Heo, P., Gu, G.M., Lee, S.-j., Rhee, K., Kim, J.: Current hand exoskeleton technologies for rehabilitation and assistive engineering. International Journal of Precision Engineering and Manufacturing 13(5), 807-824 (2012)

17. Borboni, A., Mor, M., Faglia, R.: Gloreha-hand robotic rehabilitation: design, mechanical model, and experiments. Journal of Dynamic Systems, Measurement, and Control 138(11), 111003 (2016)

18. Buch, E., Weber, C., Cohen, L.G., Braun, C., Dimyan, M.A., Ard, T., Mellinger, J., Caria, A., Soekadar, S., Fourkas, A., et al.: Think to move: a neuromagnetic brain-computer interface (bci) system for chronic stroke. Stroke 39(3), 910-917 (2008)

19. Nycz, C.J., Bützer, T., Lambercy, O., Arata, J., Fischer, G.S., Gassert, R.: Design and characterization of a lightweight and fully portable remote actuation system for use with a hand exoskeleton. IEEE Robotics and Automation Letters 1(2), 976-983 (2016)

20. Sabantina, L., Kinzel, F., Ehrmann, A., Finsterbusch, K.: Combining 3d printed forms with textile structures-mechanical and geometrical properties of multi-material systems. In: IOP Conference Series: Materials Science and Engineering, vol. 87, p. 012005 (2015). IOP Publishing

21. Rivera, M.L., Moukperian, M., Ashbrook, D., Mankoff, J., Hudson, S.E.: Stretching the bounds of 3d printing with embedded textiles. In: Proceedings of the $2017 \mathrm{CHI}$ Conference on Human Factors in Computing Systems, pp. 497-508 (2017). ACM

22. Auras, R., Harte, B., Selke, S.: An overview of polylactides as packaging materials. Macromolecular bioscience 4(9), 835-864 (2004)

23. Calì, J., Calian, D.A., Amati, C., Kleinberger, R., Steed, A., Kautz, J., Weyrich, T.: 3d-printing of non-assembly, articulated models. ACM Transactions on Graphics (TOG) 31(6), 130 (2012)

24. Lambercy, O., Dovat, L., Gassert, R., Burdet, E., Teo, C.L., Milner, T.: A haptic knob for rehabilitation of hand function. IEEE Transactions on Neural Systems and Rehabilitation Engineering 15(3), 356-366 (2007)

25. Kamper, D.G., Rymer, W.Z.: Quantitative features of the stretch response of extrinsic finger muscles in hemiparetic stroke. Muscle \& Nerve: Official Journal of the American Association of Electrodiagnostic Medicine 23(6), 954-961 (2000)

26. Kamper, D.G., Fischer, H.C., Cruz, E.G., Rymer, W.Z.: Weakness is the primary contributor to finger impairment in chronic stroke. Archives of physical medicine and rehabilitation 87(9), 1262-1269 (2006)

27. Goiriena, A., Retolaza, I., Cenitagoya, A., Martinez, F., Riano, S., Landaluze, J.: Analysis of bowden cable transmission performance for orthosis applications. In: Mechatronics, 2009. ICM 2009. IEEE International Conference On, pp. 1-6 (2009). IEEE

28. Carlson, L.E., Veatch, B.D., Frey, D.D.: Efficiency of prosthetic cable and housing. JPO: Journal of Prosthetics and Orthotics 7(3), 96-99 (1995)

29. Budynas, R.G., Nisbett, J.K.: Shigley's Mechanical Engineering Design, 10 edn. McGraw-Hill Education, New York, NY (2011)

30. Pfurtscheller, G., Neuper, C.: Motor imagery and direct brain-computer communication. Proceedings of the IEEE 89(7), 1123-1134 (2001)

31. Silva, C.R., de Araújo, R.S., Albuquerque, G., Moioli, R.C., Brasil, F.L.: Interfacing brains to robotic devices-a vrpn communication application. In: XXVI Brazilian Congress on Biomedical Engineering, pp. 597-603 (2019). Springer

32. Zuniga, J., Katsavelis, D., Peck, J., Stollberg, J., Petrykowski, M., Carson, A., Fernandez, C.: Cyborg beast: a low-cost 3d-printed prosthetic hand for children with upper-limb differences. BMC research notes 8(1), 10 (2015)

33. Fix-it: (2019). http://usefix.it/

34. Randazzo, L., Iturrate, I., Perdikis, S., Millán, J.d.R.: mano: A wearable hand exoskeleton for activities of daily living and neurorehabilitation. IEEE Robotics and Automation Letters 3(1), 500-507 (2018)

35. Arata, J., Ohmoto, K., Gassert, R., Lambercy, O., Fujimoto, H., Wada, I.: A new hand exoskeleton device for rehabilitation using a three-layered sliding spring mechanism. In: Robotics and Automation (ICRA), 2013 IEEE International Conference On, pp. 3902-3907 (2013). IEEE

36. OpenEEG: (2019). http://openeeg.sourceforge.net/doc/

37. Balasubramanian, S., Garcia-Cossio, E., Birbaumer, N., Burdet, E., Ramos-Murguialday, A.: Is emg a viable alternative to bci for detecting movement intention in severe stroke? IEEE Transactions on Biomedical Engineering 65(12), 2790-2797 (2018)

38. Ribeiro, P.R.A., Lima Brasil, F., Witkowski, M., Shiman, F., Cipriani, C., Vitiello, N., Carrozza, M.C., Soekadar, S.R.: Controlling assistive machines in paralysis using brain waves and other biosignals. Advances in Human-Computer Interaction 2013 (2013)

Figures

Figure 13 design of HERO. Isometric view at left and top view at right. 
Figure 2 Fabric samples. (A) T1 sample. (B) T2 sample. (C) T3 sample.

Figure 3 Printing tests. (A) All the three samples attached to the heated bed. Samples T3 (B), T2 (C) and T1 (D) were analysed in a stereoscopic microscope.

Figure 4 Cable-driven mechanism. The absence of a mandrel to wrap the cable can cause it to flex under compression. This mechanism is not dimensionally efficient by virtue of its long length.

Figure 5 Concertina mechanism. This mechanism consists of 6 limiters that prevent the cables to flex. The distance between the limiters is reduced as cables are pushed.

Figure 6 Actuator's 3D model. (1) DC motor; (2) universal couplings for $6 \mathrm{~mm}$ (for the lead scew) and $4 \mathrm{~mm}$ (for the DC motor); (3) nut; (4) concertina mechanism; (5) stainless steel lead screw M6x1; (6) actuator base; (7) bearing mandrel; (8) ball bearing; (9) conduit entries (* auxiliary view of a model adaptation for use with only one cable); (10) conduit fixation screw; (11) pin holes to fix micro switch bases; (12) nut pins groove; (13) fixation bracket; (14) micro switch base; (15) honeycomb structure.

Figure 7 Static scheme for cable tension estimation. $T$ is the estimated cable tension, $T_{\text {req }}$ is the required cable tension, and $F_{\mathrm{f}}$ is the Bowden cable friction force.

Figure 8 Final prototype of the exoskeleton's glove.

Figure 9 Mean force per actuator voltage supply.

Figure 10 The HERO attached to the body. The actuators were attached to the right lower limb - on the same side of the exoskeleton's glove - only for illustration. Real users should have the actuators attached to their healthy lower limbs.

Figure 11 Bits sent to Arduino Uno according to OV algorithm outputs in trials with a right arrow (MI of the right hand). The green mark represents the moment in which the cross appears on the screen. The yellow mark represents the moment in which the cue to class 2 appears on the screen. The value " 0 " means the actuators are braked, and the value "1" means the actuators are activated. Upper graph: Trial recorded between 40 and 48 seconds. Bottom graphic: Trial recorded between 187 and 195 seconds.

Figure 12 2D-trajectories of finger flexion movements. The blue curve represents the trajectory of the index fingertip during the execution of an active/natural movement - performed by the subject. The orange curve represents the passive movement - performed by the exoskeleton. 
Figures
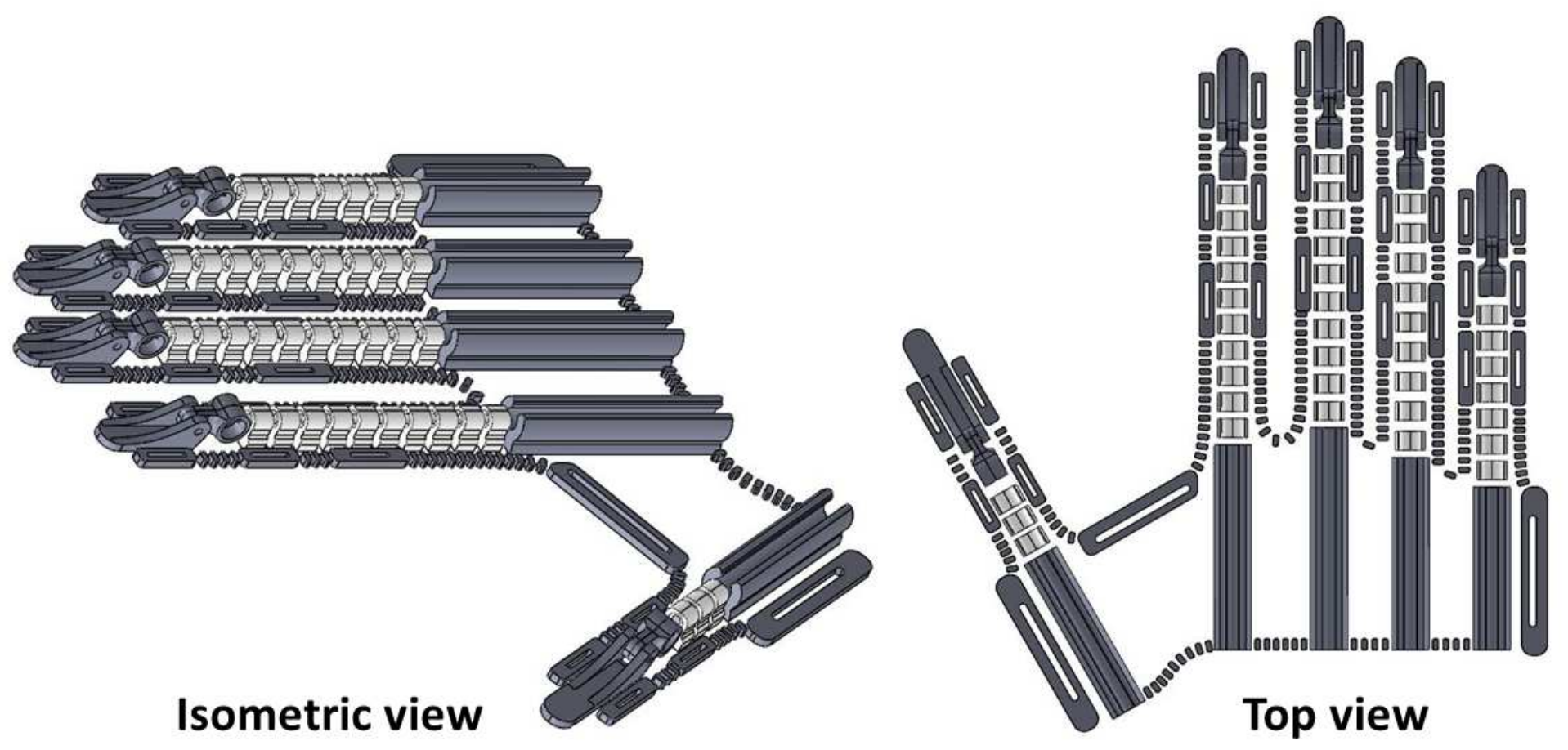

Figure 1

3D design of HERO. Isometric view at left and top view at right. 


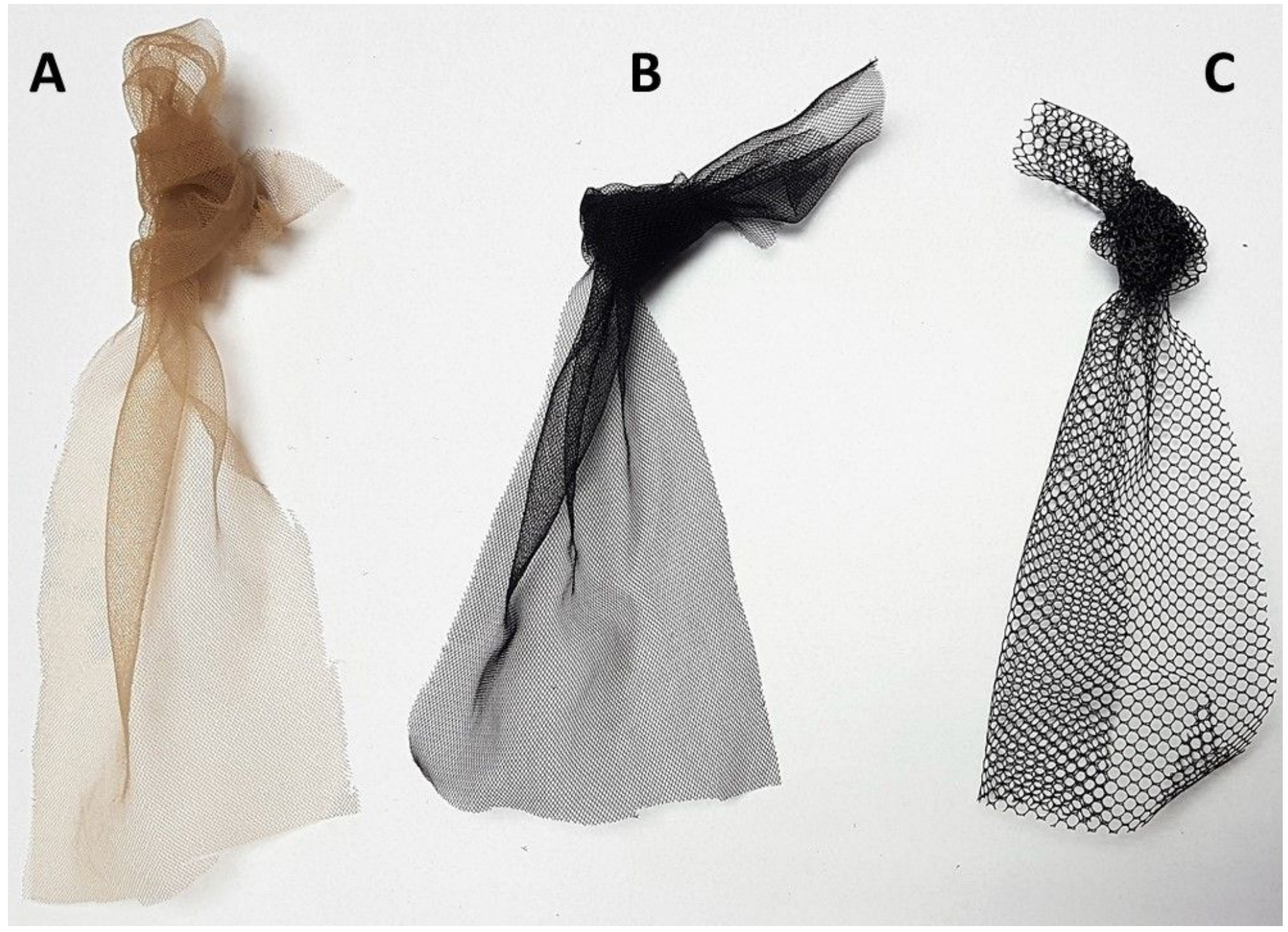

Figure 2

Fabric samples. (A) T1 sample. (B) T2 sample. (C) T3 sample. 

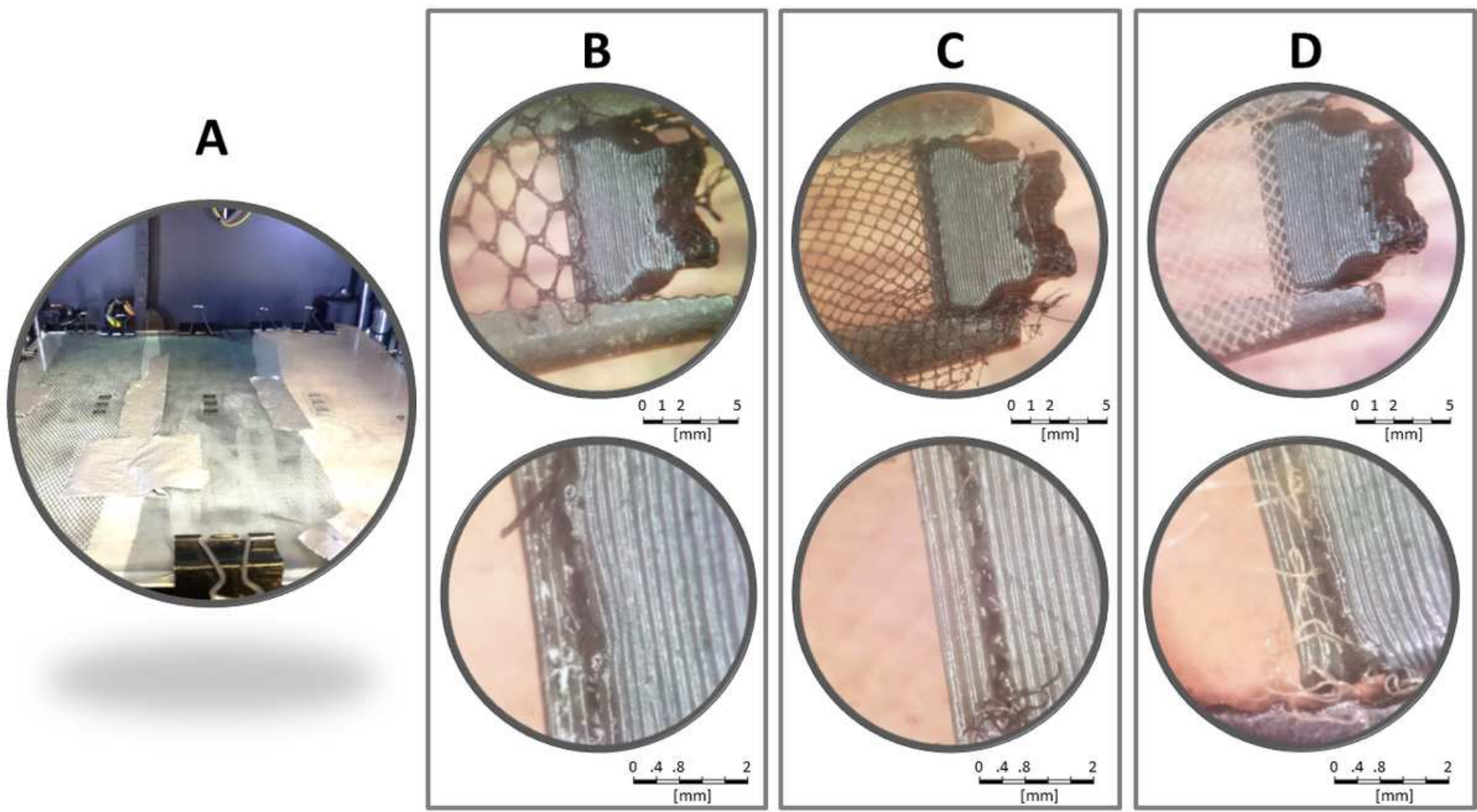

Figure 3

Printing tests. (A) All the three samples attached to the heated bed. Samples T3 (B), T2 (C) and T1 (D) were analysed in a stereoscopic microscope.

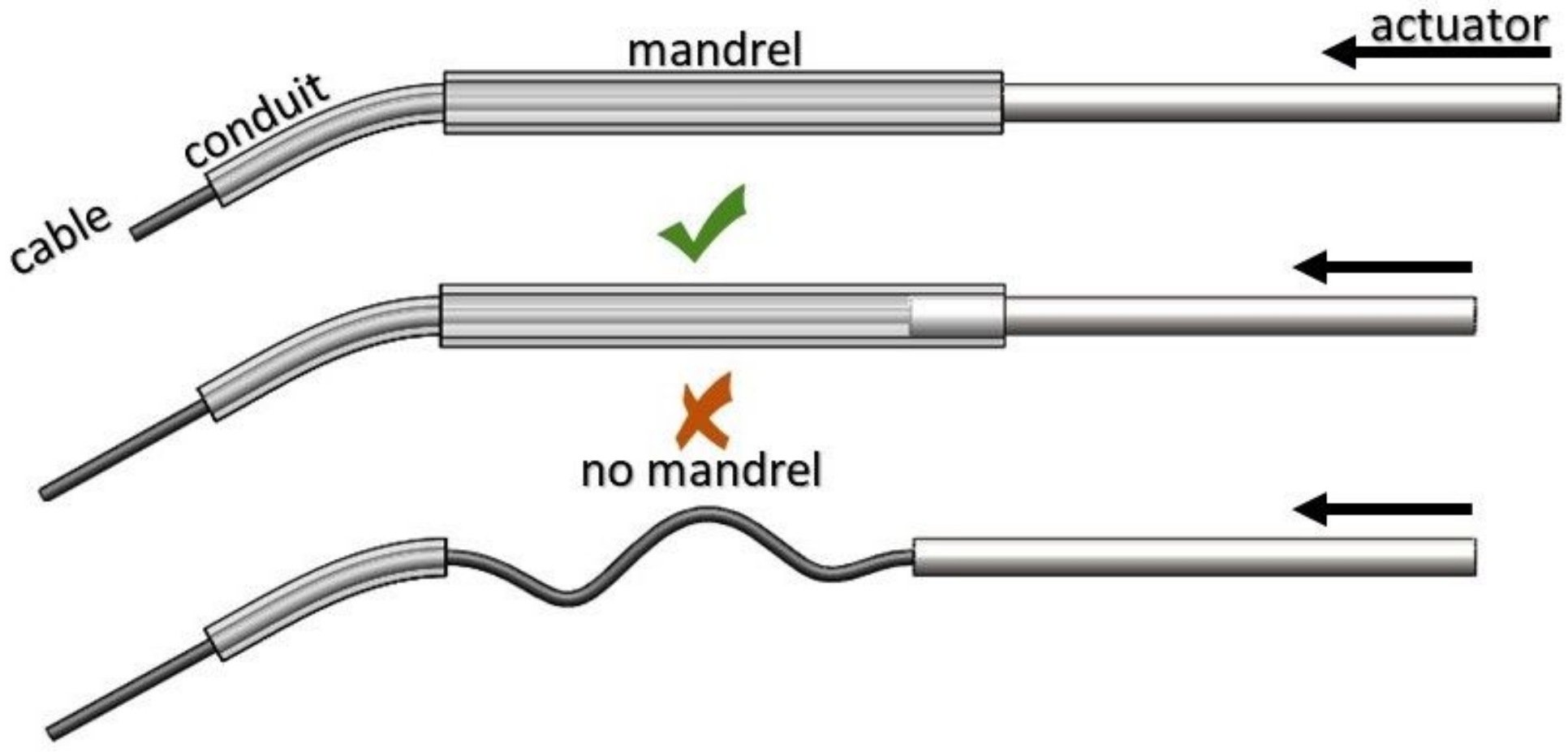

Figure 4 
Cable-driven mechanism. The absence of a mandrel to wrap the cable can cause it to ex under compression. This mechanism is not dimensionally e『cient by virtue of its long length.

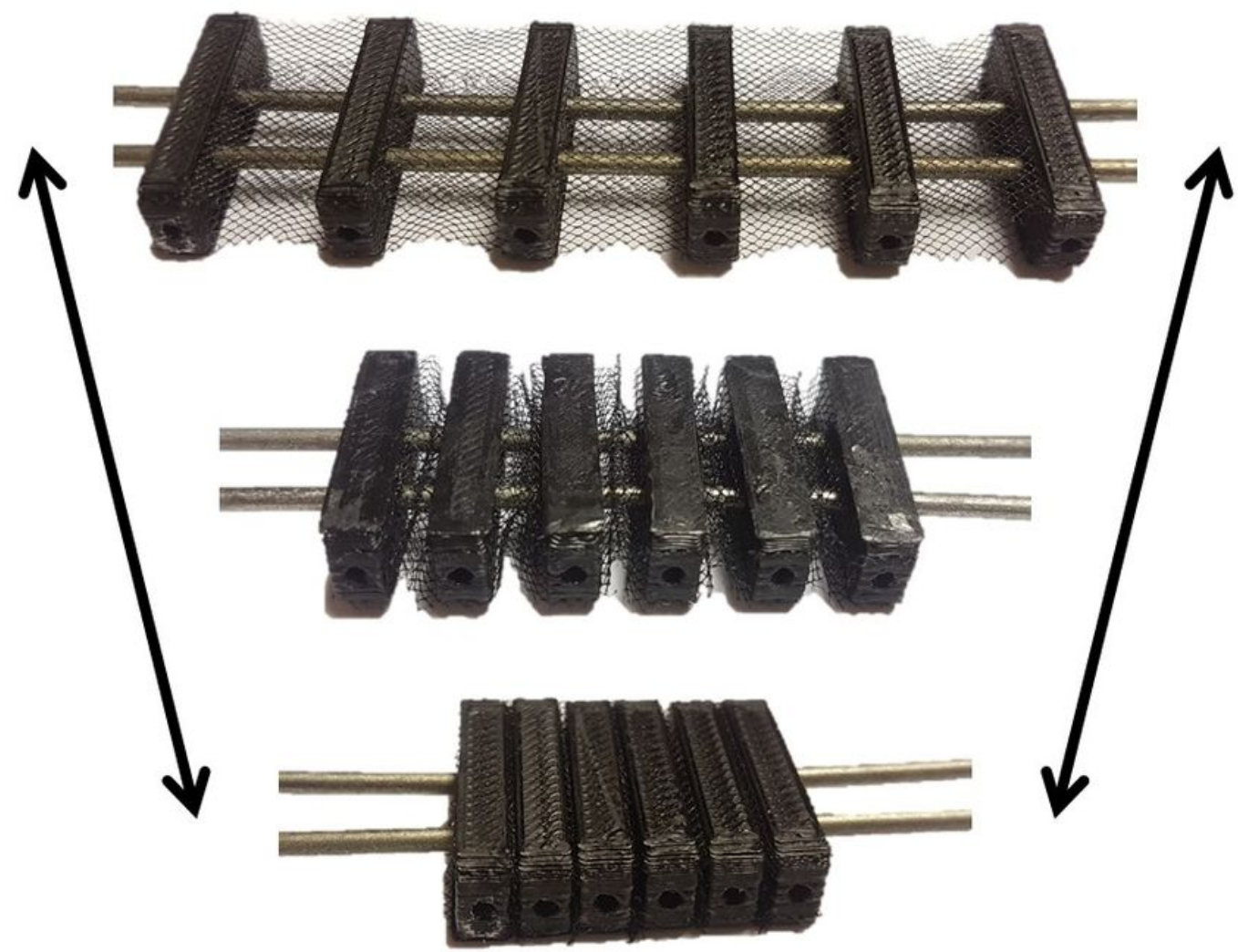

\section{Figure 5}

Concertina mechanism. This mechanism consists of 6 limiters that prevent the cables to ex. The distance between the limiters is reduced as cables are pushed.

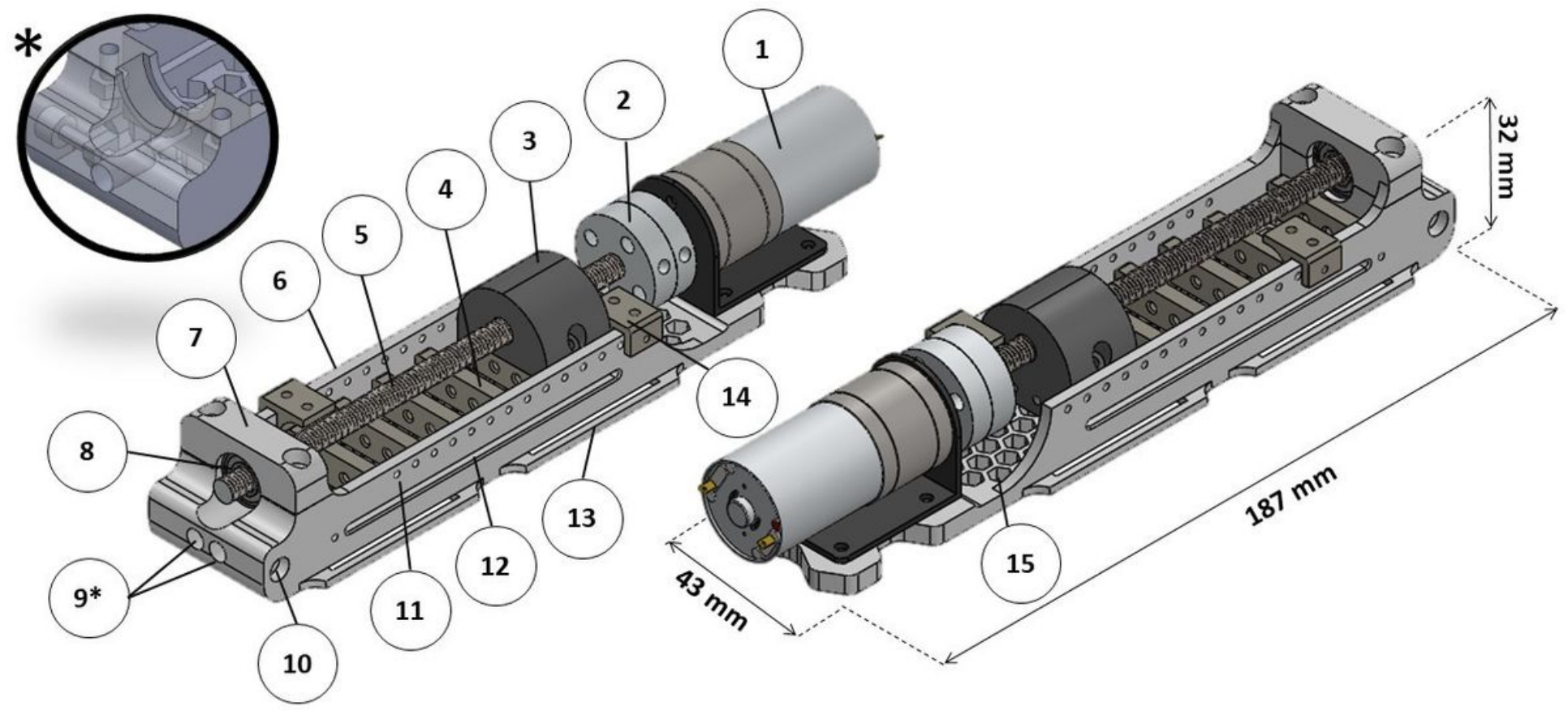

Figure 6 
Actuator's 3D model. (1) DC motor; (2) universal couplings for $6 \mathrm{~mm}$ (for the lead scew) and $4 \mathrm{~mm}$ (for the DC motor); (3) nut; (4) concertina mechanism; (5) stainless steel lead screw M6x1; (6) actuator base; (7) bearing mandrel; (8) ball bearing; (9) conduit entries (*auxiliary view of a model adaptation for use with only one cable); (10) conduit xation screw; (11) pin holes to x micro switch bases; (12) nut pins groove; (13) xation bracket; (14) micro switch base; (15) honeycomb structure.

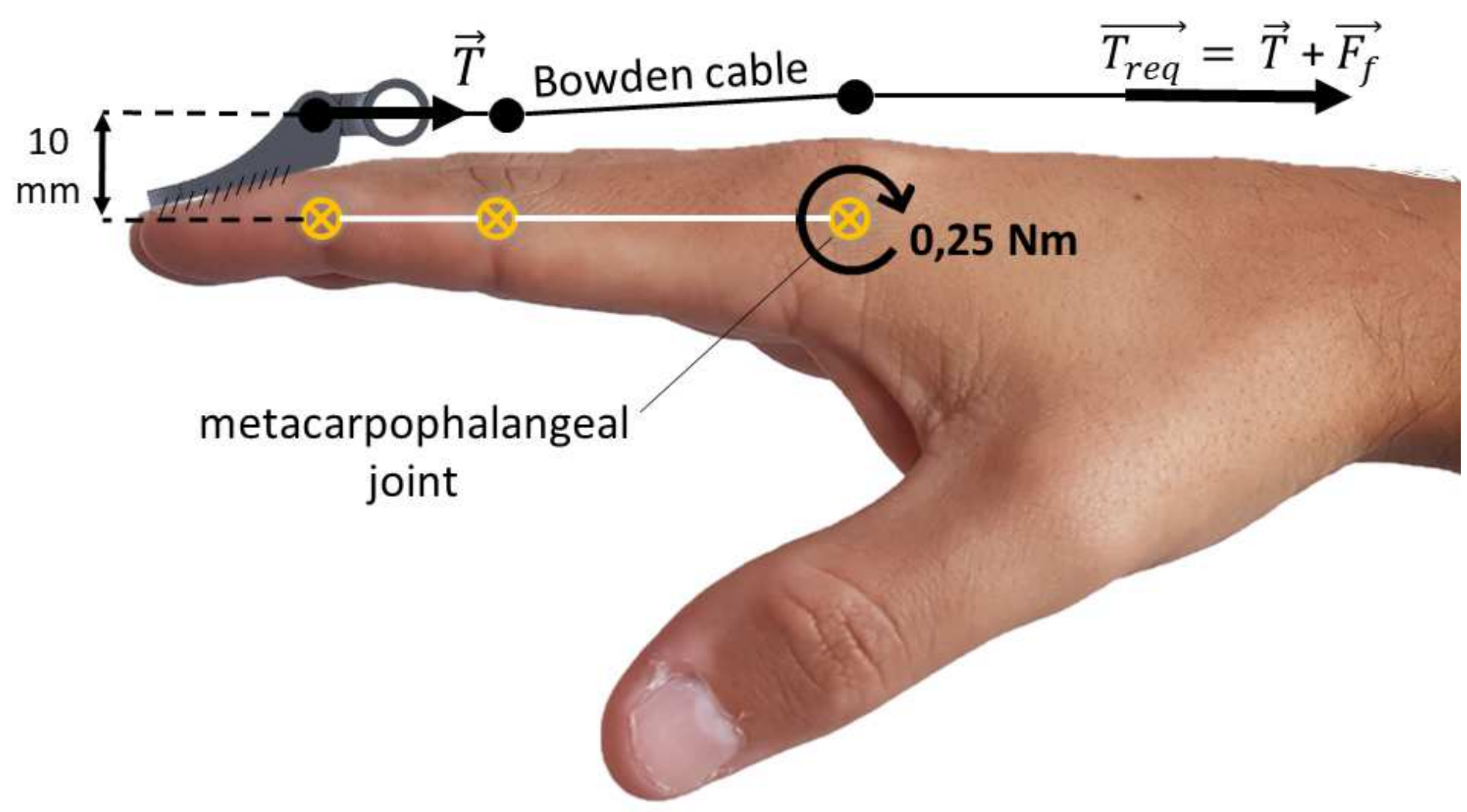

\section{Figure 7}

Static scheme for cable tension estimation. $T$ is the estimated cable tension, Treq is the required cable tension, and $\mathrm{Ff}$ is the Bowden cable friction force. 

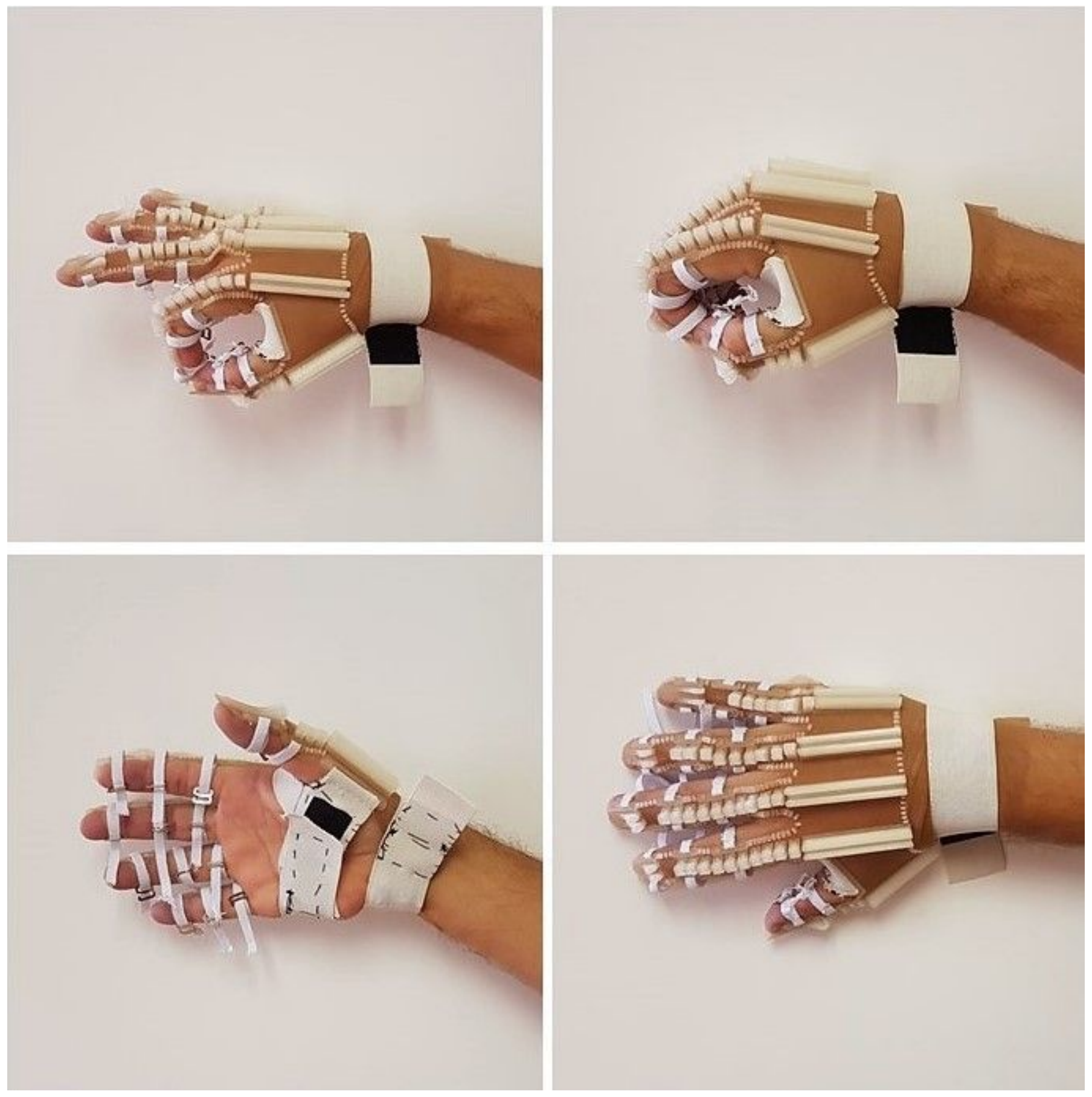

Figure 8

Final prototype of the exoskeleton's glove. 


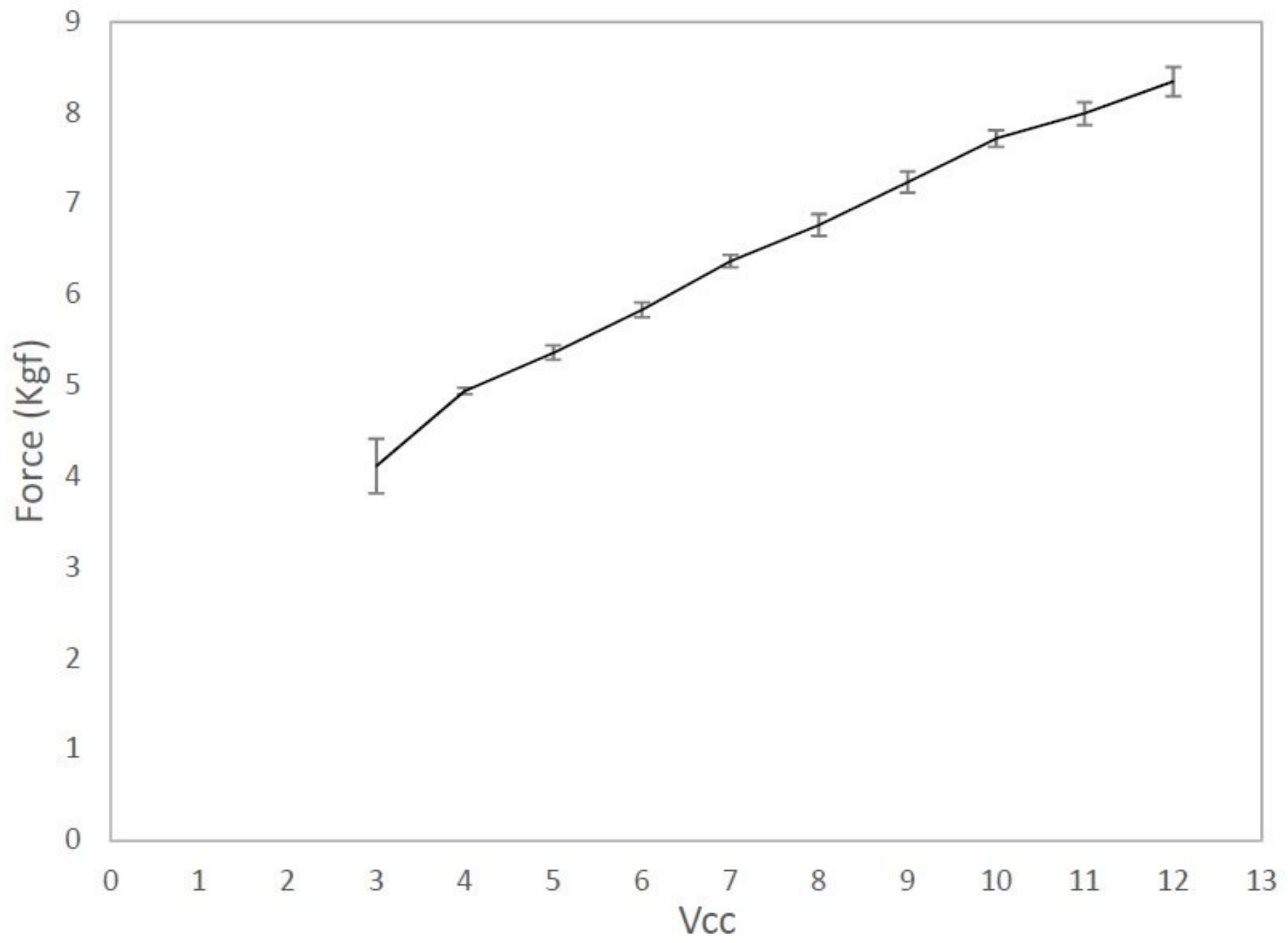

Figure 9

Mean force per actuator voltage supply. 

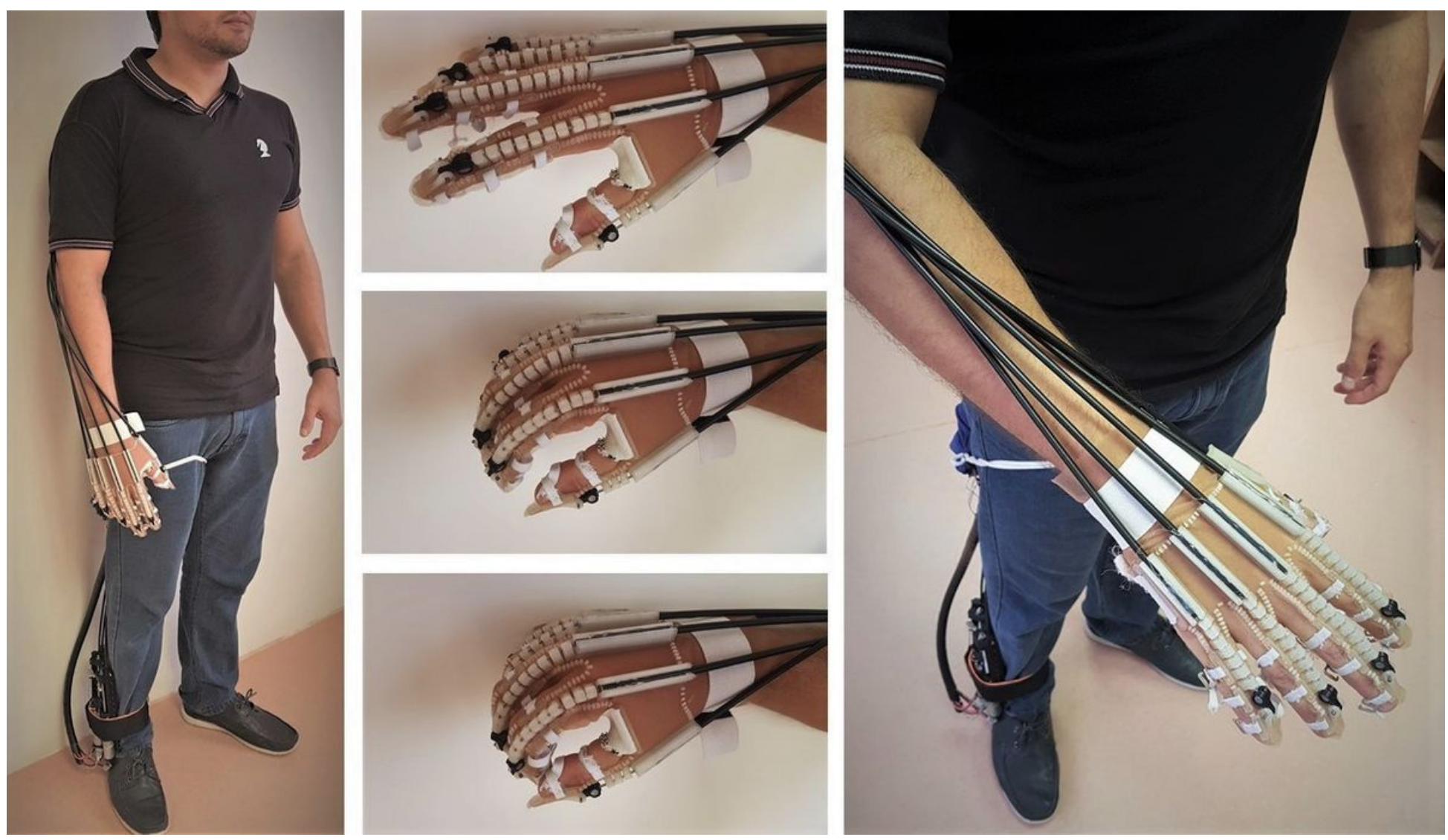

Figure 10

The HERO attached to the body. The actuators were attached to the right lower limb | on the same side of the exoskeleton's glove | only for illustration. Real users should have the actuators attached to their healthy lower limbs.

\section{Bits sent to Arduino Uno}

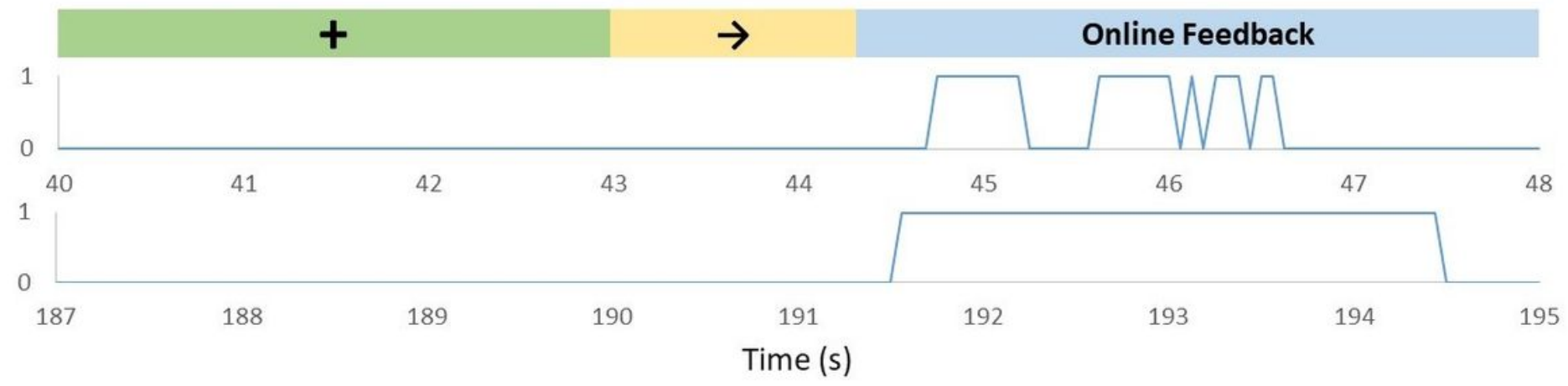

\section{Figure 11}

Bits sent to Arduino Uno according to OV algorithm outputs in trials with a right arrow (MI of the right hand). The green mark represents the moment in which the cross appears on the screen. The yellow mark represents the moment in which the cue to class 2 appears on the screen. The value 10 " means the actuators are braked, and the value $\backslash 1 "$ means the actuators are activated. Upper graph: Trial recorded between 40 and 48 seconds. Bottom graphic: Trial recorded between 187 and 195 seconds. 


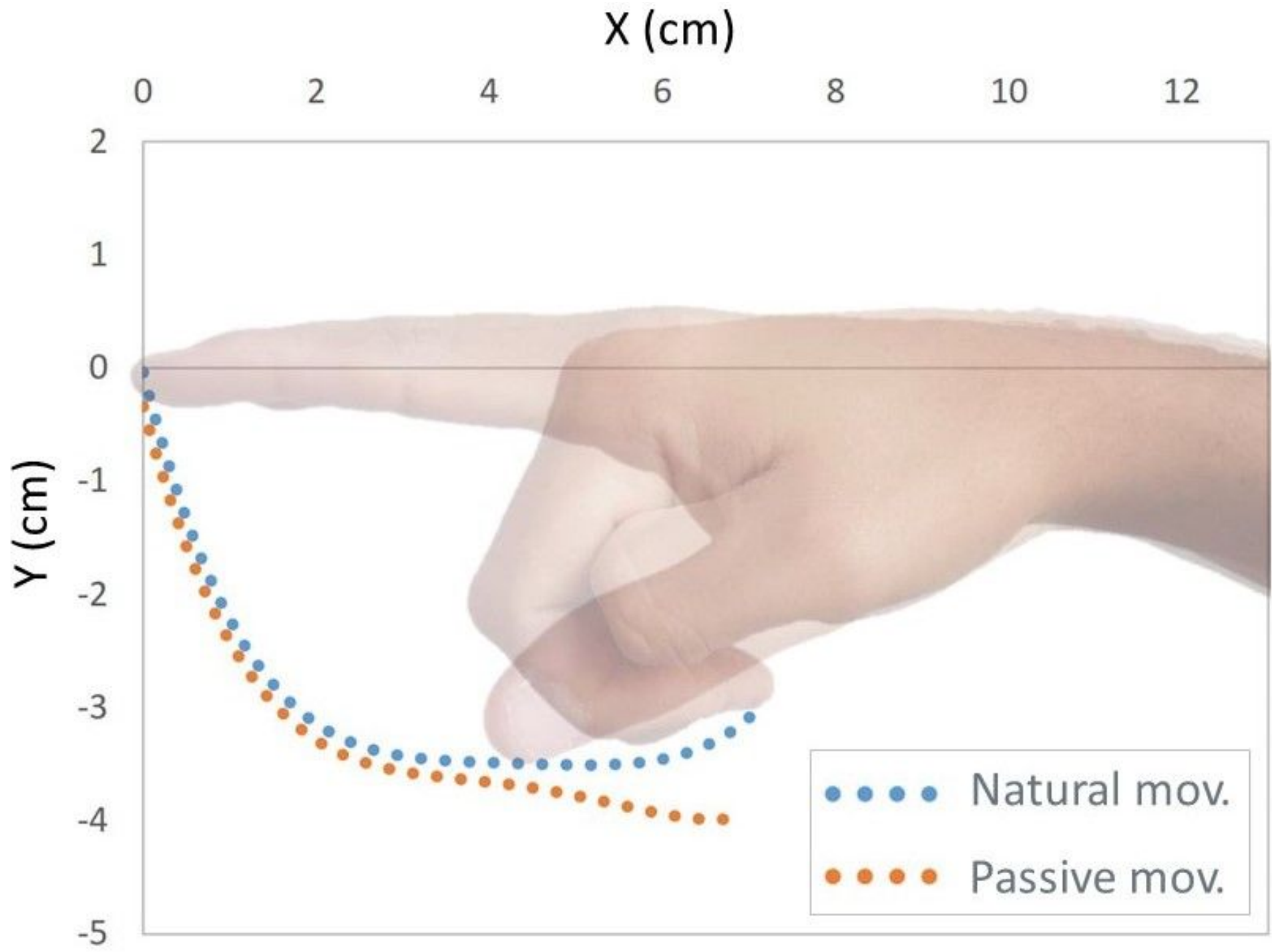

Figure 12

2D-trajectories of nger exion movements. The blue curve represents the trajectory of the index ngertip during the execution of an active/natural movement I performed by the subject. The orange curve represents the passive movement I performed by the exoskeleton.

\section{Supplementary Files}

This is a list of supplementary files associated with this preprint. Click to download.

- Additionalfile1Listofmaterialcosts.pdf 